\title{
Fighting Acne for the Fighting Forces
}

\author{
Catherine Brahe, MD; Kristopher Peters, DO
}

\section{PRACTICE POINTS}

- Acne is a common disease that may cause considerable physical and psychological morbidity. Numerous therapies are available, each with their respective risks and benefits.

- Military servicemembers face unique challenges in the management of acne due to operational and medical readiness considerations.

- Less conventional treatments such as photodynamic therapy and pulsed dye laser may be available to military servicemembers.

- Pulsed dye laser is an effective alternative treatment of acne, especially in an age of growing antibiotic resistance. their assigned duties. Treatments themselves also may be limiting; for instance, aircrew members who are taking oral doxycycline, tetracycline, or erythromycin may be grounded (ie, temporarily removed from duty) during and after therapy to monitor for side effects. Minocycline is considered unacceptable for aviators and is completely restricted for use due to risk for central nervous system side effects. Isotretinoin is restricted in aircrew members, submariners, and divers. If initiated, isotretinoin requires grounding for the entire duration of therapy and up to 3 months following treatment. Normalization of triglyceride levels and slit-lamp ocular examination also must take place prior to return to full duty, which may lead to additional grounding time. Well-established topical and oral treatments not impacting military duty are omitted from this review.

\section{Antibiotics}

Minocycline-Minocycline carries a small risk for development of systemic lupus erythematosus and other autoimmune treatment-emergent adverse effects. It has known gastrointestinal tract side effects, and long-term use also can lead to bluish discoloration of the skin. ${ }^{1}$ Systemic minocycline is restricted in aircrew members due to its risk for central nervous system side effects, including light-headedness, dizziness, and vertigo. ${ }^{2-5}$

A topical formulation of minocycline recently was developed and approved by the US Food and Drug Administration as a means to reduce systemic adverse effects. This $4 \%$ minocycline foam has thus far been safe and well tolerated, with adverse events reported in less than $1 \%$ of study participants. ${ }^{1,6}$ In addition, topical minocycline was shown in a recent phase 3 study to notably reduce inflammatory lesion counts when compared to control vehicles at as early as 3 weeks. ${ }^{7}$ Topical minocycline may emerge as a viable treatment option for active-duty servicemembers in the future.

Doxycycline-Doxycycline is not medically disqualifying. Even so, it may still necessitate grounding for a period of time while monitoring for side effects. ${ }^{4}$ Doxycycline can lead to photosensitivity, which could be difficult to

Dr. Brahe is from Naval Medical Center Portsmouth, Virginia, and currently is serving with 3rd Battalion 6th Marines, Camp Lejeune, North Carolina. Dr. Peters is from the Department of Dermatology, Madigan Army Medical Center, Tacoma, Washington.

The authors report no conflict of interest.

The views expressed are those of the authors and do not reflect the official views or policy of the US Department of Defense.

Correspondence: Catherine Brahe, MD (Catherine.a.brahe.mil@mail.mil). 
tolerate for active-duty personnel training in sunny climates. Fortunately, uniform regulations and personal protective equipment requirements provide cover for most of the body surfaces aside from the face, which is protected by various forms of covers. If the patient tolerates the medication well without considerable side effects, he/she may be returned to full duty, making doxycycline an acceptable alternative to minocycline in the military population.

Sarecycline-This novel compound is a tetracycline-class antibiotic with a narrower spectrum of activity, with reduced activity against enteric gram-negative bacteria. It has shown efficacy in reducing inflammatory and noninflammatory acne lesions, including lesions on the face, back, and chest. Common adverse side effects are nausea, headache, nasopharyngitis, and vomiting. Vestibular and phototoxic adverse effects were reported in less than $1 \%$ of patients. ${ }^{1,8}$ The US Food and Drug Administration approved sarecycline as a once-daily oral formulation for moderate to severe acne vulgaris, the first new antibiotic to be approved for the disease in the last 40 years. Sarecycline is not mentioned in any US military guidelines with regard to medical readiness and duty status; however, given its lack of vestibular side effects and narrower activity spectrum, it may become another acceptable treatment option in the military population.

\section{Isotretinoin}

Isotretinoin is well established as an excellent treatment of acne and stands alone as the only currently available medication that alters the disease course and prevents relapse in many patients. Nearly all patients on isotretinoin experience considerable mucocutaneous dryness, and up to $25 \%$ of patients on high-dose isotretinoin develop myalgia. ${ }^{9}$ Isotretinoin causes serious retinoid embryopathy, requiring all patients to be enrolled in the iPLEDGE program (https://www.ipledgeprogram.com/iPledgeUI/home.u) and to use 2 methods of contraception during treatment. Although it is uncommon to have notable elevations in lipids and transaminases during treatment with isotretinoin, routine laboratory monitoring generally is performed until the patient reaches steady dosing.

Isotretinoin is not permitted for use in active aircrew members, submariners, or divers. Servicemembers pursuing isotretinoin therapy are removed from their duty and are nondeployable for the entirety of their treatment course and several months after completion. ${ }^{4,5}$

\section{Photodynamic Therapy}

Aminolevulinic acid and photodynamic therapy (ALA-PDT) has been successfully used in the management of acne. ${ }^{10}$ In addition to inducing selective damage to sebaceous glands, it has been proposed that PDT also destroys Propionibacterium acnes and reduces keratinocyte shedding and immunologic changes that play key roles in the development of acne. ${ }^{10}$

A recent randomized controlled trial comparing the efficacy of ALA-PDT vs adapalene gel plus oral doxycycline for treatment of moderate acne included 46 patients with moderate inflammatory acne. ${ }^{10}$ Twenty-three participants received 2 sessions (spaced 2 weeks apart) of $20 \%$ ALA incubated for 90 minutes before red light irradiation with a fluence of $37 \mathrm{~J} / \mathrm{cm}^{2}$, and the other 23 received $100 \mathrm{mg} / \mathrm{d}$ of oral doxycycline plus adapalene gel $0.1 \%$. By 6-week followup, there was a significantly higher reduction in total lesions within the PDT group $(P=.038)$, which was sustained at the secondary 12 -week follow-up $(P=.026)$. There was a $79 \%$ total reduction of lesions in the ALA-PDT group vs $67 \%$ in the doxycycline plus adapalene group. ${ }^{10}$

Although some studies have shown promise for PDT as an emerging treatment option for acne, further research is needed. A 2016 systematic review of the related literature determined that although 20\% ALA-PDT with red light was more effective than lower concentrations of ALA and also more effective than ALA-PDT with blue lightwhich offered no additional benefit when compared with blue light alone-high-quality evidence on the use of PDT for acne is lacking overall. ${ }^{11}$ At the time of the review, there was little certainty as to the usefulness of ALA-PDT with red or blue light as a standard treatment for individuals with moderate to severe acne. A 2019 review by Marson and Baldwin ${ }^{12}$ echoed this sentiment, recommending more stringently designed studies to elucidate the true role of PDT as a monotherapy or adjunctive treatment of acne.

\section{Pulsed Dye Laser}

Pulsed dye laser (PDL) was first shown to be a potential therapy for acne by Seaton et al, ${ }^{13}$ who conducted a smallscale, randomized, controlled trial with 41 patients, each assigned to either a single PDL treatment or a sham treatment. Patients were re-evaluated at 12 weeks, measuring acne severity by the Leeds revised acne grading system and taking total lesion counts. Acne severity $(P=.007)$ and total lesion counts $(P=.023)$ were significantly improved in the treatment group, with a $53 \%$ reduction in total lesion count following a single PDL treatment. ${ }^{13}$

In 2007, a Spanish study described use of PDL every 4 weeks for a total of 12 weeks in 36 patients with mild to moderate acne. Using lesion counts as their primary outcome measure, the investigators found results similar to those from Seaton et $\mathrm{al}_{1}{ }^{13}$ with a $57 \%$ decrease in active lesions. ${ }^{14}$ Others still have found similar outcomes. A 2009 study of 45 patients with mild to moderate acne compared patients treated with PDL every 2 weeks for 12 weeks to patients receiving either topical therapy or chemical peels with $25 \%$ trichloroacetic acid. At 12 weeks, they noted the best results were in the PDL group. ${ }^{15}$

Karsai et $\mathrm{al}^{16}$ compared PDL as an adjuvant treatment of acne to proven treatment with clindamycin plus benzoyl peroxide gel. Eighty patients were randomized to topical therapy plus PDL or topical therapy alone and were followed at 2 and 4 weeks after the initial treatment. Although both groups showed improvement as measured by inflammatory lesion count and dermatology life quality index, there was no statistically significant difference noted between groups. ${ }^{16}$ 
A, Demonstration of new and active inflammatory and cystic acne lesions, most noticeable surrounding the mouth and forehead, following 3 months of twice-daily doxycycline therapy. B, Reduction in active acne lesions and improvement in overall erythema and irritation following 3 treatments with the $595-\mathrm{nm}$ pulsed dye laser (spot size, $10 \mathrm{~mm}$; fluence, $7 \mathrm{~J} / \mathrm{cm}^{2}$; pulse width, 6 milliseconds) spaced 4 weeks apart.
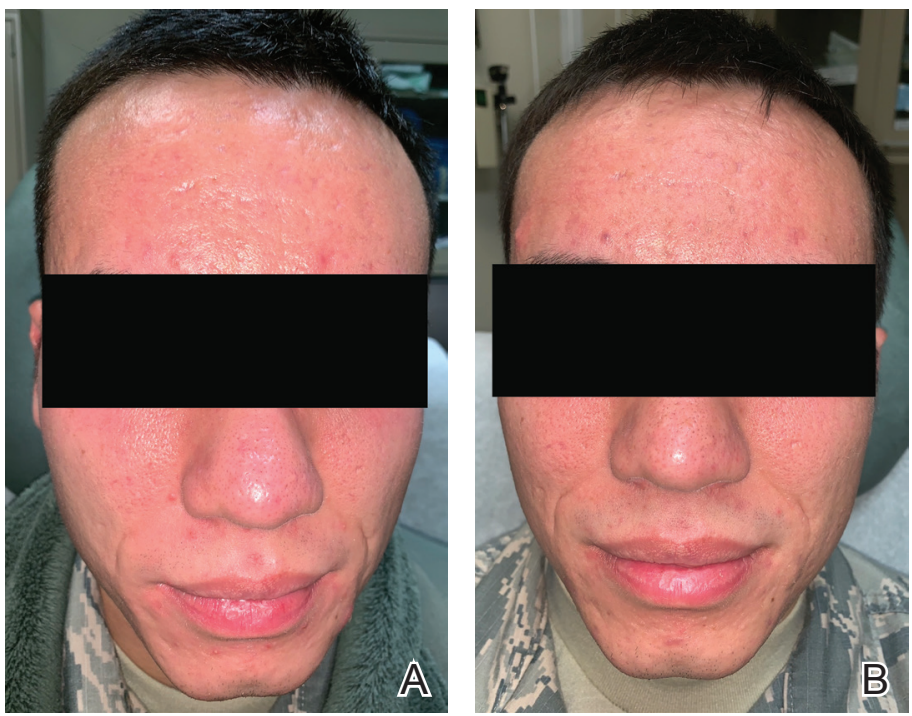

\section{Case Report}

A 24-year-old active-duty male servicemember was referred to the dermatology department for evaluation of treatment-resistant nodulocystic scarring acne. Prior to his arrival to dermatology, he had completed 2 weeks of isotretinoin before discontinuation due to notable mood alteration. Following the isotretinoin, he was then switched to doxycycline $100 \mathrm{mg}$ twice daily, which he trialed for 3 months. Even on the antibiotic, the patient continued to develop new pustules and cysts, prompting referral to dermatology for additional treatment options (Figure, A). All of the previous topical and oral medications had been discontinued at the current presentation.

The patient received 3 treatments with the 595-nm PDL (spot size, $10 \mathrm{~mm}$; fluence, $7 \mathrm{~J} / \mathrm{cm}^{2}$; pulse width, 6 milliseconds) spaced 4 weeks apart. At each treatment, fewer than 10 total inflammatory lesions were treated, including inflammatory papules, pustules, and nodules. Nodular lesions were treated with 2 pulses. After each treatment, the patient reported that all treated lesions resolved within 2 days (Figure, B). Subsequent treated lesions all occurred at previously uninvolved sites.

\section{Final Thoughts}

Antibiotic resistance is a known and growing problem throughout the medical community. In 2013, the US Centers for Disease Control and Prevention reported that dermatologists prescribe more antibiotics than any other specialty. ${ }^{17}$ Aside from antibiotic stewardship, systemic antibiotics come with various considerations when selecting ideal acne treatment regimens in military populations, as they are either medically disqualifying or lead to temporary grounding status. Numerous guidelines on acne have recommended limiting the use of antibiotics, instead pursuing alternative therapies such as spironolactone, oral contraceptives, or isotretinoin..$^{9,18}$ Both spironolactone and oral contraceptives work well via antiandrogenic and antisebogenic properties; however, these therapies are limited to female patients only, who make up a minority of patients in the active-duty military setting. Isotretinoin is highly effective in the treatment of acne, but it requires grounding for the entirety of treatment and for months afterward, which comes at great personal and financial costs to servicemembers and their commanders due to limited-duty status and inability to deploy.

Given the operational constraints with isotretinoin and the continual rise of antibiotic resistance, PDL appears to be a safe and effective alternative therapy for acne. In our case, the patient had complete resolution of active inflammatory lesions after each of his treatments. He had no adverse effects and tolerated the treatments well. We report this case here to highlight the use of PDL as an effective therapy for spot treatment in patients limited by personal or operational constraints and as a means to reduce antibiotic use in the face of a growing tide of antibiotic resistance.

\section{REFERENCES}

1. Kircik LH. What's new in the management of acne vulgaris. Cutis. 2019;104:48-52.

2. US Department of the Army. Standards of medical fitness. https://armypubs.army.mil/epubs/DR_pubs/DR_a/pdf/web /ARN8673_AR40_501_FINAL_WEB.pdf. Published June 27, 2019. Accessed June 23, 2020.

3. US Department of the Air Force. Medical examinations and standards. http://aangfs.com/wp-content/uploads/2012/10/AFI-48-123-Medical -Examination-Standards.pdf. Published January 29, 2013. Accessed June 23, 2020.

4. US Navy Aeromedical Reference and Waiver Guide. Navy Medicine website. https://www.med.navy.mil/sites/nmotc/nami/arwg /Documents/WaiverGuide/Complete_Waiver_Guide.pdf. Published September 4, 2019. Accessed June 17, 2020.

5. Burke KR, Larrymore DC, Cho S. Treatment considerations for US military members with skin disease. Cutis. 2019:103:329-332.

6. Gold LS, Dhawan S, Weiss J, et al. A novel topical minocycline foam for the treatment of moderate-to-severe acne vulgaris: results of 2 randomized, double-blind, phase 3 studies. J Am Acad Dermatol. 2019;30:168-177.

7. Raoof J, Hooper D, Moore A, et al. FMX101 4\% topical minocycline foam for the treatment of moderate to severe acne vulgaris: efficacy and safety from a phase 3 randomized, double-blind, CONTINUED ON PAGE 22 


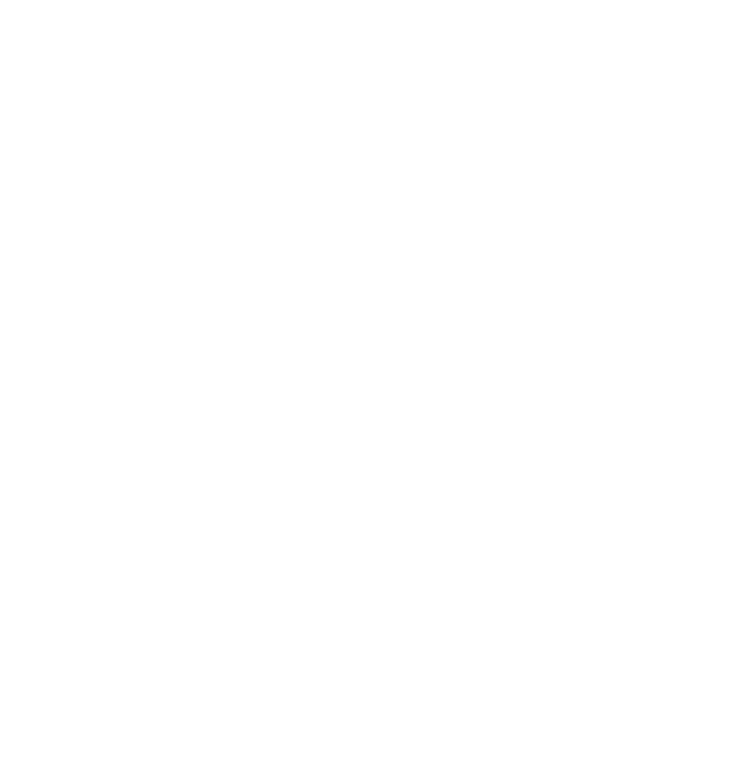

MILITARY DERMATOLOGY

CONTINUED FROM PAGE 20

vehicle-controlled study. Poster presented at: 2018 Fall Clinical Dermatology Conference; October 18-21, 2018; Las Vegas, NV.

8. Moore A, Green LJ, Bruce S, et al. Once-daily oral sarecycline $1.5 \mathrm{mg} / \mathrm{kg} /$ day is effective for moderate to severe acne vulgaris; results from two identically designed, phase 3, randomized, double-blind clinical trials. J Drugs Dermatol. 2018;17:987-996.

9. Barbieri JS, Spaccarelli N, Margolis DJ, et al. Approaches to limit systemic antibiotic use in acne: systemic alternatives, emerging topical therapies, dietary modification, and laser and light-based treatments. J Am Acad Dermatol. 2019;80:538-549.

10. Nicklas C, Rubio R, Cardenas C, et al. Comparison of efficacy of aminolaevulinic acid photodynamic therapy vs. adapalene gel plus oral doxycycline for treatment of moderate acne vulgaris—a simple, blind, randomized, and controlled trial. Photodermatol Photoimmunol Photomed. 2019;35:3-10.

11. Barbaric J, Abbott R, Posadzki P, et al. Light therapies for acne [published online September 27, 2016]. Cochrane Database Syst Rev. doi:10.1002/14651858.CD007917.pub2.

12. Marson JW, Baldwin HE. New concepts, concerns, and creations in acne. Dermatol Clin. 2019;37:1-9.
13. Seaton ED, Charakida A, Mouser PE, et al. Pulsed-dye laser treatment for inflammatory acne vulgaris: randomised controlled trial. Lancet Lond Engl. 2003;362:1347-1352.

14. Harto A, Garcia-Morales I, Belmar P, et al. Pulsed dye laser treatment of acne. study of clinical efficacy and mechanism of action. Actas Dermosifiliogr. 2007;98:415-419.

15. Leheta TM. Role of the 585-nm pulsed dye laser in the treatment of acne in comparison with other topical therapeutic modalities. J Cosmet Laser Ther Off Publ Eur Soc Laser Dermatol. 2009;11:118-124.

16. Karsai S, Schmitt L, Raulin C. The pulsed-dye laser as an adjuvant treatment modality in acne vulgaris: a randomized controlled singleblinded trial. Br J Dermatol. 2010;163:395-401.

17. Centers for Disease Control and Prevention. Outpatient antibiotic prescriptions-United States. annual report 2013. https://www.cdc.gov/antibiotic-use/community/pdfs/Annual -ReportSummary_2013.pdf. Accessed June 23, 2020.

18. Zaenglein AL, Pathy AL, Schlosser BJ, et al. Guidelines of care for the management of acne vulgaris. J Am Acad Dermatol. 2016; 74:945-973.e33. 